\title{
ON EXPONENTIAL DICHOTOMY IN BANACH SPACES
}

\author{
Mihail Megan and Petre Preda
}

\begin{abstract}
In this paper we study the exponential dichotomy property for linear systems, the evolution of which can be described by a semigroup of class $C_{0}$ on a Banach space. We define the class of $(p, q)$ dichotomic semigroups and establish the connections between the dichotomy concepts and admissibility property of the pair $\left(L^{p}, L^{q}\right)$ for linear control systems. The obtained results are generalizations of well-known results of W.A. Coppel, J.L. Massera and J.J. Schäffer, K.J. Palmer.
\end{abstract}

\section{Introduction}

In Perron's classical paper on stability ([8]) a central concern is the relationship, for linear differential equations, between the condition that the nonhomogeneous equation has some bounded solution for every bounded "second member", on the one hand, and a certain form of conditional stability of the solutions of the homogeneous equation on the other. This idea was later extensively developed among others by Massera and Schäffer in [4] and Coppel in [2].

The extension of the bounded input, bounded output criteria of Perron for the case of linear control systems has been studied by several authors [4], [5], [6], [8]. The relationship between the conditional input-output stability and the exponential dichotomy for the case of a finitedimensional linear control system is considered by Palmer in [7].

The aim of this paper is to study the exponential dichotomy property

Received 10 November 1980. 
for linear systems, the evolution of which can be described by a semigroup of class $C_{0}$ on a Banach space. Using a fundamental inequality established in [4] we define the concept of $(p, q)$ dichotomic semigroup and give a sufficient condition for exponential dichotomy of a large class of such semigroups. We also give a proof for the equivalence between the exponential dichotomy of a $C_{0}$ semigroup $T(t)$ and $\left(L^{p}, L^{q}\right)$ admissibility property for the case of a linear control system

$$
x\left(t, x_{0}, u\right)=T(t) x_{0}+\int_{0}^{t} T(t-s) B u(s) d s .
$$

The case $T(t)=\exp (A t)$, where $A$ is a bounded linear operator on a finite dimensional space has been considered by Palmer in [7].

\section{Definitions and terminology}

Let $T(t)$ be a $C_{0}$ semigroup on a separable Banach space $X$. Consider the control process described by the following integral model,

$$
\left(T, B, U_{p}\right) x\left(t, x_{0}, u\right)=T(t) x_{0}+\int_{0}^{t} T(t-s) B u(s) d s,
$$

under the following standard assumptions: $x(t, \cdot, \cdot)$ belongs to $X$; $u \in U_{p}=L^{p}\left(R_{+}, U\right)$ where $R_{+}=[0, \infty)$ and $U$ is also a Banach space; $B \in L(U, X)$ (the space of bounded linear operators from $U$ to $X$ ); finally $x_{0} \in X$.

Here $u_{p}$ is the Banach space of all $U$-valued, strongly measurable functions $u$ defined almost everywhere on $R_{+}$such that

$$
\|u\|_{p}=\left(\int_{0}^{\infty}\|u(s)\|^{p} d s\right)^{1 / p}<\infty \text {, if } p<\infty,
$$

and

$$
\|u\|_{\infty}=\underset{s \geq 0}{\operatorname{ess} \sup }\|u(s)\|<\infty \text {, if } p=\infty \text {. }
$$

We also denote 


$$
x_{p}=I^{p}\left(R_{+}, X\right) \text { and } p^{\prime}= \begin{cases}\infty & \text {, if } p=1, \\ 1 & , \text { if } p=, \\ p /(p-1) & , \text { if } 1<p<\infty .\end{cases}
$$

Let $X_{1}, X_{2}$ be two closed complemented subspaces of $X$ such that

$$
X=X_{1} \oplus X_{2}
$$

If we denote by $P_{1}$ a projection along $X_{2}$ (that is, $\operatorname{Ker} P_{1}=X_{2}$ ) then $P_{1} \in L\left(X, X_{1}\right), P_{1}^{2}=P_{1}$ and $P_{2}=I-P_{1}$ is a projection along $X_{1}$ with analogous properties.

We shall denote $T_{1}(t)=T(t) P_{1}$ and $T_{2}(t)=T(t) P_{2}$.

DEFINITION 2.1. The subspace $X_{1}$ induces

(i) an exponential dichotomy for the semigroup $T(t)$ if there exist constants $N>0, v>0$ such that

$$
\left\|T_{1}(t) x\right\| \leq N e^{-\nu t_{1}}\left\|P_{1} x\right\|
$$

and

$$
\left\|T_{2}(t) x\right\| \geq N e^{v t}\left\|P_{2} x\right\|
$$

for all $t \geq 0$ and $x \in X$;

(ii) a $(p, q)$ dichotomy (where $1 \leq p, q \leq \infty)$ for the semigroup $T(t)$ if there exists $N>0$ such that

$$
\begin{aligned}
& \left\|T_{1}(\cdot) x\right\|_{L^{q}[t+\delta, \infty)}+\left\|T_{2}(\cdot) x\right\|_{L^{q}[0, t]} \leq N \delta{ }^{(1 / p)-2}\|T(\cdot) x\|_{L^{1}[t, t+\delta]} \\
& \quad \text { for all } t \in 0, \delta>0 \text { and } x \in X_{k}, k=1,2 .
\end{aligned}
$$

REMARK 2.1. If $X_{1}$ induces an exponential dichotomy for $T(t)$ then

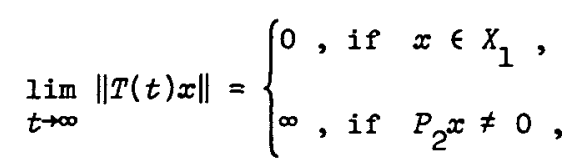

and hence $X_{1}=\left\{x \in X: \lim _{t \rightarrow \infty} T(t) x=0\right\}$. 
REMARK 2.2. If $X_{1}$ induces an exponential dichotomy for $T(t)$ then

$$
X_{1}=\left\{x \in X: T(\cdot) x \in X_{q}\right\}
$$

where $1 \leq q \leq \infty$.

DEFINITION 2.2. The $C_{0}$ semigroup $T(t)$ is said to be exponentially dichotomic $((p, q)$ dichotomic) if there exists a closed complemented subspace $X_{1}$ which induces an exponential dichotomy $((p, q)$ dichotómy) for $T(t)$.

DEFINITION 2.3. Let $1 \leq p, q \leq \infty$. The pair $\left(u_{p}, x_{q}\right)$ is admissible for $\left(T, B, u_{p}\right)$ if for every $u \in u_{p}$ there exists $x_{u} \in X$ such that $x\left(\cdot, x_{u}, u\right) \in X_{q}$.

Now let us note four assumptions which will be used at various times.

ASSUMPTION 1. We say that the semigroup $T(t)$ satisfies Assumption 1 if for every $q \geq 1$ the set

$$
X_{1}=\left\{x \in X: T(\cdot) x \in X_{q}\right\}
$$

is a closed complemented subspace.

ASSUMPTION 2. The semigroup $T(t)$ satisfies Assumption 2 if for every $t_{0} \geq 0$ there exist $t_{1} \geq t_{0}$ and $m_{1}>0$ such that

$$
\left\|T_{2}\left(t_{1}\right) x_{0}\right\| \geq m\left\|P_{2} x_{0}\right\|
$$

for all $x_{0} \in X$.

ASSUMPTION 3. The system $\left(T, B, U_{p}\right)$ satisfies Assumption 3 if the range of $B$ is of second category in $X$.

ASSUMPTION 4. The semigroup $T(t)$ satisfies Assumption 4 if

$$
T_{1}(t) \neq 0 \text { for every } t \geq 0 \text { and any } x \in X_{1}, x \neq 0 .
$$

\section{Preliminary results}

We state the following

LEMMA 3.1. If $T(t)$ is a $C_{0}$ semigrowp then there exist $M>1$, 
$\omega>0$ such that

$$
\begin{aligned}
& \text { (i) }\|T(t)\| \leq M e^{\omega t} \text { for alz } t \geq 0 ; \\
& \text { (ii) }\|T(t) x\| \leq M e^{\omega \delta}\|T(s) x\| \text { for } a l l \quad \delta>0 \text { and } \\
& 0 \leq s \leq t \leq s+\delta ; \\
& \text { (iii) } \delta\|T(t) x\| \leq M e^{\omega \delta} \cdot \int_{t-\delta}^{t}\|T(s) x\| d s \text { for any } \delta>0 \text { and } \\
& t \geq \delta ; \\
& \text { (iv) } \int_{t}^{t+\delta}\|T(s) x\| d s<M e^{\omega \delta} \cdot\|T(t) x\| \text { for all } t \geq 0 \text { and } \delta>0 .
\end{aligned}
$$

Proof. It is well known (see [1], pp. 165-166) that if

$$
\omega \geq \varlimsup_{t \rightarrow \infty} \frac{\ln \|T(t)\|}{t}=\inf _{t>0} \frac{\ln \|T(t)\|}{t}=\omega_{0}<\infty
$$

then there exists $M \geq 1$ such that (i) holds.

The inequalities $(i i)-(i v)$ follow immediately from (i) and the semigroup property.

LEMMA 3.2. Suppose that Assumption 1 holds and let $x_{2}$ be $a$ complementary subspace of $x_{1}$. If $\left(u_{p}, x_{q}\right)$ is admissible for $\left(T, B, u_{p}\right)$ then there exists $N>0$ such that for every $u \in u_{p}$ there is an unique $x_{2}(u) \in X_{2}$ with the properties:

(i) $x\left(\cdot, x_{2}(u), u\right) \in X_{q}$, and

(ii) $\left\|x\left(\cdot, x_{2}(u), u\right)\right\|_{q} \geqq N\left\|_{u}\right\|_{p}$.

Proof. Let $u \in u_{p}$. Then by admissibility of $\left(u_{p}, x_{q}\right)$ for $\left(T, B, u_{p}\right)$ there exists $x_{0} \in X$ such that

$$
x\left(\cdot, x_{0}, u\right) \in X_{q} .
$$

If we denote by $x_{k}=P_{k} x_{0}(k=1,2)$ then from the definition of $X_{1}$ we have that $x\left(\cdot, x_{1}, 0\right) \in X_{q}$ and hence 


$$
x\left(\cdot, x_{2}, u\right)=x\left(\cdot, x_{0}, u\right)-x\left(\cdot, x_{1}, 0\right) \in \mathrm{X}_{q} .
$$

It follows that for every $u \in u_{p}$ there is $x_{2}(u)=P_{2} x_{0}, x_{2}$ with the property $(i)$.

If we suppose that there exist $x_{2}^{\prime}, x_{2}^{\prime \prime} \in X_{2}$ such that $x\left(\cdot, x_{2}^{\prime}, u\right) \in X_{q}$ and $x\left(\cdot, x_{2}^{\prime \prime}, u\right) \in X_{q}$ then

$$
x\left(\cdot, x_{2}-x_{2}^{\prime \prime}, u\right)=x\left(\cdot, x_{2}^{\prime}-x_{2}^{\prime \prime}, 0\right)=T(\cdot)\left(x_{2}^{\prime}-x_{2}^{\prime \prime}\right) \in X_{q}
$$

and hence

$$
x_{2}^{\prime}-x_{2}^{\prime \prime} \in X_{1} \cap X_{2}=\{0\}
$$

which shows that $x_{2}^{\prime}=x_{2}^{\prime \prime}$.

Let $\Lambda: u_{p} \rightarrow x_{q}$ be the operator defined by

$$
\Lambda u=x\left(\cdot, x_{2}(u), u\right) \text {. }
$$

It is easy to see that $\Lambda$ is linear (from uniqueness of $x_{2}(u)$ ). Property $(i i)$ is equivalent with the statement that $\Lambda$ is a bounded operator. From the closed graph theorem it is sufficient to prove that $\Lambda$ is closed.

Let $u_{n} \rightarrow u$ in $u_{p}$ and $u_{n} \rightarrow x$ in $x_{q}$. Let $\left(u_{n_{k}}\right)$ be a subsequence of $\left(u_{n}\right)$ such that $u_{n_{k}} \rightarrow u$ almost everywhere.

Because we may suppose that $x(\cdot)$ is continuously, we have that

$$
\lim _{k \rightarrow \infty} x_{2}\left(u_{n_{k}}\right)=\lim _{k \rightarrow \infty}\left(\Lambda u_{n_{k}}\right)(0)=x(0) \in X_{2}
$$

and hence

$$
\begin{array}{r}
x(t)=\lim _{k \rightarrow \infty}\left(T(t) x_{2}\left(u_{n_{k}}\right)+\int_{0}^{t} T(t-s) B u_{n_{k}}(s) d s\right) \\
=T(t) x(0)+\int_{0}^{t} T(t-s) B u(s) d s=x(t, x(0), u) .
\end{array}
$$

From $x(\cdot) \in X_{q}$, we have that $x(0)=x_{2}(u)$ and hence 


$$
x(t)=x\left(t, x_{2}(u), u\right)=(\Lambda u)(t) \text { for all } t \geq 0
$$

LEMMA 3.3. If $T(t)$ is $(p, q)$ dichotomic with $(p, q) \neq(1, \infty)$ then there exists a function $\eta: R_{+} \rightarrow R_{+}$with $\lim _{t \rightarrow \infty} \eta(t)=0$ and such that for all $\delta_{0}>0$ and $\delta>\delta_{0}$ we have

$$
\begin{aligned}
& \text { (i) } \int_{t}^{t+\delta}\left\|T_{1}(s) x\right\| d s \leq n\left(\delta_{0}\right) \cdot \int_{t_{0}}^{t_{0}+\delta}\left\|T_{1}(s) x\right\| d s \text {, for alz } \\
& t_{0} \geq 0, \quad t \geq t_{0}+\delta_{0} \text { and alz } x \in X ; \text { and } \\
& \text { (ii) } \int_{t_{0}}^{t_{0}+\delta}\left\|T_{2}(s) x\right\| d s \leq n\left(\delta_{0}\right) \cdot \int_{t}^{t+\delta}\left\|T_{2}(s) x\right\| d s \text { for alz } \\
& t_{0} \geq 0, \quad t \geq t_{0}+2 \delta_{0} \text { and } x \in X .
\end{aligned}
$$

Proof. Let $\delta>\delta_{0}>0$ and let $n$ be a positive integer such that $n \delta_{0} \leq \delta<(n+1) \delta_{0}$.

If we denote by $\delta_{1}=\delta / n$ then from $t>t_{0}+\delta_{0}$ and $s=t_{0}+k \delta_{1}$, $k=0,1, \ldots, n-1$, by $(p, q)$ dichotomy of $T(t)$ and Hölder's inequality we have

$$
\begin{aligned}
\int_{8+t-t_{0}}^{s+t-t_{0}+\delta_{1}}\left\|T_{1}(\tau) x\right\| d \tau \leq \delta_{1}^{1 / q^{\prime}} \cdot\left(\int_{8+\delta_{0}}^{\infty}\left\|T_{1}(\tau) x\right\|^{q} d \tau\right)^{1 / q} \\
\leq\left(2 \delta_{0}\right)^{1 / q} \cdot \int_{s}^{s+\delta_{0}}\left\|T_{1}(\tau) x\right\| d \tau<n\left(\delta_{0}\right) \cdot \int_{s}^{s+\delta_{1}}\left\|T_{1}(\tau) x\right\| d \tau,
\end{aligned}
$$

where

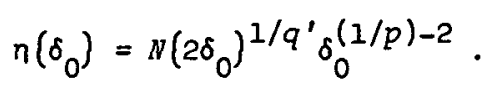

Taking $s=t_{0}+k \delta_{1}, k=0,1,2, \ldots, n-1$ and adding we obtain

$$
\int_{t}^{t+\delta}\left\|T_{1}(\tau) x\right\| d \tau=\int_{t}^{t+n \delta_{1}}\left\|T_{1}(\tau) x\right\| d \tau \leq n\left(\delta_{0}\right) \cdot \int_{t_{0}}^{t_{0}}\left\|T_{1}(\tau) x\right\| d \tau
$$


and hence (i) is proved.

Let $t \geq t_{0}+2 \delta_{0}$ and $s=t_{0}+k \delta_{1}$ with $k=0,1, \ldots, n-1$. Then as before we have

$$
\begin{aligned}
& \int_{s}^{s+\delta_{1}}\left\|T_{2}(\tau) x\right\| d \tau \leq \delta_{1}^{l / q^{\prime}} \cdot\left(\int_{s}^{s+\delta_{1}}\left\|T_{2}(\tau) x\right\|^{q} d \tau\right)^{1 / q} \\
& \leq i^{1 / q^{\prime}} \cdot\left(\int_{0}^{s+t-t_{0}}\left\|T_{2}(\tau) x\right\|^{q} d \tau\right)^{1 / q} \leq n\left(\delta_{0}\right) \cdot \int_{s+t-t_{0}}^{s+t-t_{0}+\delta_{0}}\left\|T_{2}(\tau) x\right\| d \tau \\
& \leq n\left(\delta_{0}\right) \cdot \int_{s+t-t_{0}}^{s+t-t_{0}+\delta_{1}}\left\|T_{2}(\tau) x\right\| d \tau
\end{aligned}
$$

and adding, we obtain the inequality (ii).

LEMMA 3.4 ([4]). Let $f: R_{+} \rightarrow R_{+}$be a function with the property that there is $\delta>0$ such that $f(t+\delta) \geq 2 f(t)$ for every $t>0$ and $2 f(t) \geq f\left(t_{0}\right)$ for all $t_{0} \geq 0$ and $t \in\left[t_{0}, t_{0}+\delta\right]$. Then there exists $v>0$ such that

$$
4 f(t) \geq e^{v\left(t-t_{0}\right)} f\left(t_{0}\right) \text { for all } t \geq t_{0} \geq 0 .
$$

The proof is immediate. Indeed, if $v=(\ln 2) / \delta$ and $n$ is the positive integer with

$$
n \delta \leq t-t_{0}<(n+1) \delta
$$

then

$$
4 f(t) \geq 2 f\left(t_{0}+n \delta\right) \geq 2^{n+1} f\left(t_{0}\right)=e^{\nu(n+1) \delta} f\left(t_{0}\right) \geq e^{\nu\left(t-t_{0}\right)} f\left(t_{0}\right) .
$$

LEMMA 3.5. If $T(t)$ is $(p, q)$ dichotomic with $(p, q) \neq(1, \infty)$ then there exists $v>0$ such that for every $\delta>0$ there is $N>0$ with

$$
\begin{aligned}
& \text { (i) } \int_{t}^{t+\delta}\left\|T_{1}(s) x\right\| d s \leq N e^{-v\left(t-t_{0}\right)}\left\|T_{1}\left(t_{0}\right) x\right\| \text {, and } \\
& \text { (ii) } \int_{t_{0}}^{t_{0}+\delta}\left\|T_{2}(s) x\right\| d s \leq N e^{-v\left(t-t_{0}\right)}\left\|T_{2}\left(t_{0}\right) x\right\| \text { for all } t \geq t_{0} \geq 0
\end{aligned}
$$


and $x \in X$.

Proof. Let $\delta>0, x \in X$ and let $\delta_{0}$ be sufficiently large such that

$$
n\left(\delta_{0}\right)<\frac{1}{2}
$$

Let $n$ be a positive integer such that $n \delta>4 \delta_{0}$ and let us consider the function $f: R_{+} \rightarrow R_{+}$defined by

$$
f(t)=\left(\int_{t}^{t+n \delta}\left\|T_{1}(s) x\right\| d s\right)^{-1}
$$

By Lemma 3.3 we obtain

$$
\int_{t_{0}+\delta_{0}}^{t_{0}+\delta_{0}+n \delta}\left\|T_{1}(s) x\right\| d s \leq n\left(\delta_{0}\right) \int_{t_{0}}^{t_{0}+n \delta}\left\|T_{1}(s) x\right\| d s \leq \frac{2}{2} \int_{t_{0}}^{t_{0}+n \delta}\left\|T_{1}(s) x\right\| d s
$$

and hence

$$
f\left(t_{0}+\delta_{0}\right) \geq 2 f\left(t_{0}\right)
$$

If $t \in\left|t_{0}, t_{0}+\delta_{0}\right|$ then

$$
\begin{aligned}
\int_{t}^{t+n \delta}\left\|T_{1}(s) x\right\| d s \leq \int_{t_{0}}^{t_{0}+\delta_{0}}\left\|T_{1}(s) x\right\| d s+\int_{t_{0}+\delta_{0}}^{t_{0}+\delta_{0}+n \delta}\left\|T_{1}(s) x\right\| d s & \leq 2 \int_{t_{0}}^{t_{0}+n \delta}\left\|T_{1}(s) x\right\| d s,
\end{aligned}
$$

which implies that

$$
2 f(t) \geq f\left(t_{0}\right) \text { for every } t \in\left[t_{0}, t_{0}+\delta_{0}\right] .
$$

From Lemma 3.4 we obtain that there exists $v>0$ such that

$$
4 f(t) \geq f\left(t_{0}\right) e^{v\left(t-t_{0}\right)} \text { for all } t \geq t_{0} \geq 0 \text {. }
$$

By the preceding inequality and Lemma 3.1 we conclude that 


$$
\begin{aligned}
\int_{t}^{t+\delta}\left\|T_{1}(s) x\right\| d s=\frac{1}{f(t)} \leq 4 e^{-\nu\left(t-t_{0}\right)} \cdot \int_{t_{0}}^{t_{0}+n \delta}\left\|T_{1}(s) x\right\| d s & \leq 4 M n \delta e^{n \omega \delta} \cdot e^{-\nu\left(t-t_{0}\right)}\left\|T_{1}\left(t_{0}\right) x\right\|
\end{aligned}
$$

for all $t \geq t_{0} \geq 0$. The inequality is proved.

From ( $i i$ ) let $g$ be the function defined by

$$
g(t)=\int_{t}^{t+n \delta}\left\|T_{2}(s) x\right\| d s .
$$

Then from inequality (ii) of Lemma 3.3 we obtain

$$
g\left(t_{0}+2 \delta_{0}\right)=\int_{t_{0}+2 \delta_{0}}^{t_{0}+2 \delta_{0}+n \delta}\left\|T_{2}(s) x\right\| d s \geq 2 \int_{t_{0}}^{t_{0}+n \delta}\left\|T_{2}(s) x\right\| d s=2 g\left(t_{0}\right)
$$

and for $t \in\left[t_{0}, t_{0}+2 \delta_{0}\right]$ we have

$$
\begin{aligned}
g\left(t_{0}\right)=\int_{t_{0}}^{t_{0}+n \delta}\left\|T_{2}(s) x\right\| d s \leq \int_{t_{0}}^{t_{0}+2 \delta} \| & \left\|T_{2}(s) x\right\| d s+\int_{t}^{t+n \delta}\left\|T_{2}(s) x\right\| d s \\
& \leq \frac{1}{2} \int_{t_{0}+2 \delta_{0}}^{t_{0}+4 \delta_{0}}\left\|T_{2}(s) x\right\| d s+g(t) \leq 2 g(t) .
\end{aligned}
$$

We may now apply Lemma 3.4 to $g$ and on account of Lemma 3.2 it follows that

$$
\begin{aligned}
& \int_{t_{0}}^{t_{0}+\delta}\left\|T_{2}(s) x\right\| d s \leq g\left(t_{0}\right) \leq 4 e^{-v\left(t-t_{0}\right)} g(t) \\
& \leq 4 M e^{n \omega \delta_{0}} e^{-\nu\left(t-t_{0}\right)} \cdot\left\|T_{2}(t) x\right\|=N e^{-\nu\left(t-t_{0}\right)}\left\|T_{2}(t) x\right\|
\end{aligned}
$$

for all $t \geq t_{0} \geq 0$.

\section{The main results}

The purpose of this section is to establish the connections between the dichotomy concepts and admissibility. 
THEOREM 4.1. Suppose that Assumption 2 holds. If the subspace $X_{1}$ indices $a(p, q)$ dichotomy with $(p, q) \neq(1, \infty)$ then $x_{1}$ also induces an exponential dichotomy for the semigroup $T(t)$.

Proof. Let $x \in X$ and $\delta>0$.

Firstly, we suppose that

$$
T_{1}(t) x \neq 0 \text { for all } t \geq 0 \text {. }
$$

From Lemmas 3.1 and 3.5 we find that

$$
\left\|T_{1}(t) x\right\| \leq M e^{\omega \delta} \cdot \int_{t-\delta}^{t}\left\|T_{1}(s) x\right\| d s \leq M N e^{\omega \delta} e^{-v t}\left\|P_{1} x\right\| \text { for all } t>\delta .
$$

Let

$$
N_{1}=\max \left\{M N e^{\omega \delta}, \sup _{t \in[0, \delta]} e^{\nu t}\|T(t)\|\right\}
$$

Then

$$
\begin{aligned}
& \qquad\left\|T_{1}(t) x\right\| \leq N_{1} e^{-v t_{\| P_{1}} \|} \text { for all } t \geq 0 \\
& \text { If there exists } t_{0}>0 \text { such that } T_{1}\left(t_{0}\right) x=0 \text { then } \\
& T_{1}(t) x=0 \text { for all } t \geq t_{0}
\end{aligned}
$$

and hence the preceding inequality holds.

Therefore, there exist $N_{1}, v>0$ such that

$$
\left\|T_{1}(t) x\right\| \leq N_{1} e^{-v t}\left\|P_{1} x\right\|
$$

for all $t \geq 0$ and $x \in X$.

Similarly, if $T_{2}(t) \neq 0$ for every $t \geq 0$ then using Assumption 2 and Lemmas 3.1 and 3.5 one obtains that there exist $\delta, m>0$ such that $\begin{aligned} m\left\|P_{2} x\right\| \leq\left\|T_{2}(\delta) x\right\| \leq \frac{M e^{\omega \delta}}{\delta} \cdot \int_{0}^{\delta}\left\|T_{2}(s) x\right\| d s \leq \frac{M N e^{\omega \delta}}{\delta} \cdot e^{-\nu t} \cdot\left\|T_{2}(t) x\right\| & \\ & \text { for all } t \geq 0 .\end{aligned}$ This yields 


$$
\left\|T_{2}(t) x\right\| \geq N_{2} e^{\nu t}\left\|P_{2} x\right\| \text { for all } t \geq 0
$$

where

$$
N_{2}=\frac{m \delta}{M N} e^{-\omega \delta}
$$

If there is $t_{0}>0$ with $T_{2}\left(t_{0}\right) x_{0}=0$ then $T_{2}(t) x=0$ for all $t \geq t_{0}$ and from Assumption 2 it follows that $P_{2} x=0$. This shows that the inequality

$$
\left\|T_{2}(t) x\right\| \geq N_{2} e^{\nu t}\left\|P_{2} x\right\|
$$

holds for all $t \geq 0$ and $x \in X$.

THEOREM 4.2. Assume that Assumptions 1, 3 and 4 hold. Then if the pair $\left(u_{p}, x_{q}\right)$ is admissible for $\left(T, B, u_{p}\right)$ then the semigroup $T(t)$ is $(p, q)$ dichotomic.

Proof. According to the more refined version of the open-mapping theorem ([3]) it follows that if $\left(T, B, u_{p}\right)$ satisfies Assumption 3 then there exist an operator $B^{+}: X \rightarrow U$ and $b>0$ such that

$$
B B^{+}=x \text { and }\left\|B^{+} x\right\| \leq b\|x\| \text { for every } x \in X \text {. }
$$

Let $t>0, \delta>0$ and $x \in X, x \neq 0$. Let $u_{t}(\cdot)$ be the input function defined by

$$
u_{t}(s)= \begin{cases}\frac{B^{+} t(s) x}{\|T(s) x\|}, & \text { if } s \in[t, t+\delta], \\ 0 & , \text { if } s \notin[t, t+\delta],\end{cases}
$$

and let $x_{t}^{0}=-f(t) P_{2} x$ where $f(t)=\int_{t}^{t+\delta} \frac{d s}{\|T(s) x\|}$ and $P_{2}$ is the projection along $X_{1}=\left\{x \in X: T(\cdot) x_{1} \in X_{q}\right\}$.

The function $u_{t} \in u_{p},\left\|u_{t}\right\|_{p} \leq b \delta^{1 / p}$ and 


$$
x\left(s, x_{t}^{0}, u_{t}\right)=\left\{\begin{array}{l}
f(t) T(s) P_{1} x, \text { if } s>t+\delta, \\
-f(t) T(s) P_{2} x, \text { if } s \leq t,
\end{array}\right.
$$

where $P_{1}=I-P_{2}$.

Hence $x\left(\cdot, x_{t}^{0}, u_{t}\right) \in X_{q}$ and by Lemma 3.2 we have that there is $N>0$ such that

$$
\left\|x\left(\cdot, x_{t}^{0}, u_{t}\right)\right\|_{q} \leq N \cdot\left\|u_{t}\right\|<N \cdot b \cdot \delta^{1 / p} .
$$

This shows that

$$
f(t)\left\|T_{2}(\cdot) x\right\|_{L^{q}[0, t]}+f(t)\left\|T_{1}(\cdot) x\right\|_{L^{q}[t+\delta, \infty)} \leq N b \delta^{1 / p} .
$$

By Schwartz's inequality we have

$$
\delta^{2}=f(t) \cdot \int_{t}^{t+\delta}\|T(s) x\| d s
$$

which implies that

$$
\left\|T_{1}(\cdot) x\right\|_{L^{q}}{ }_{[t+\delta, \infty)}+\left\|T_{2}(\cdot) x\right\|_{L}{ }_{[0, t]} \leq N b \delta^{(1 / p)-2} \cdot \int_{t}^{t+\delta}\|T(s) x\| d s .
$$

The theorem is proved.

COROLLARY 4.3. Assume that Assumptions 1-4 hold. Then if the pair $\left(u_{p}, x_{q}\right)$ with $(p, q) \neq(1, \infty)$ is admissible for $\left(T, B, u_{p}\right)$ then the semigroup $T(t)$ is exponentially dichotomic.

\section{References}

[1] A.V. Balakrishnan, Applied functional analysis (Applications of Mathematics, 3. Springer-Verlag, New York, Heidelberg, Berlin, 1976). 
[2] W.A. Coppe1, Dichotomies in stability theory (Lecture Notes in Mathematics, 629. Springer-Verlag, Berlin, Heidelberg, New York, 1978).

[3] Einar Hille, Ralph S. Phillips, Functional analysis and semi-groups, revised edition (American Mathematical Society Colloquium Publications, 31. American Mathematical Society, Providence, Rhode Island, 1957).

[4] José Luis Massera, Juan Jorge Schäffer, Linear differential equations and function spaces (Pure and Applied Mathematics, 21. Academic Press, New York and London, 1966).

[5] M. Megan, "On exponential stability of linear control systems in Hilbert spaces", An. Univ. Timisoaro Ser. Sti. Mat. 14 (1976), 125-130.

[6] Mihail Megan, "On the input-output stability of linear controllable systems", Canad. Math. Buzl. 21 (1978), 187-195.

[7] K.J. Palmer, "Two linear systems criteria for exponential dichotomy", Ann. Mat. Pura Appl. (to appear).

[8] Oskar Perron, "Die Stabilitätsfrage bei Differentialgleichungen", Math. Z. 32 (1930), 703-728.

[9] L.M. Silverman and B.D.O. Anderson, "Controllability, observability and stability of linear systems", SIAM J. Control Optim. 6 (1968), 121-130.

Department of Mathematics,

University of Timişoara,

1900 - Timişoara,

RS Romania. 